\title{
EL APORTE DE LA EDUCACIÓN Y LA CAPACITACIÓN PARA EL TRABAJO EN LA INDUSTRIALIZACIÓN DE ANTIOQUIA- COLOMBIA
}

\author{
Albenis María Monterroza Ríos \\ ITM (Medellín) e I.E. Jorge Eliécer \\ gaitánalbenis692@gmail.com
}

Recibido: $28 / 01 / 2018$

Aceptado: $12 / 05 / 2018$

\section{RESUMEN}

El proceso industrial colombiano, inmerso en el modelo de industrialización por sustitución de importaciones -ISI-, ocurrido entre 1945 y 1980, provocó e incentivó cambios sociotécnicos en el país, especialmente, en zonas de alta industrialización como el departamento de Antioquia y el área circundante de su capital -Medellín, Bello, Envigado, Itagüí-. Estos cambios se dieron a nivel educativo, social, económico, urbanístico, cultural y territorial, entre otros. El presente artículo pretende establecer cuál fue el aporte en el campo de la educación al proceso industrial, desde la capacitación para el trabajo que estimuló cambios socioculturales con la apertura de nuevos centros educativos que formaron a los empresarios, ingenieros, obreros y toda la mano de obra necesaria para asumir los retos del proceso industrial en esta época.

Palabras claves: Industrialización- Educación - modelo ISI - Antioquia

\section{THE CONTRIBUTION OF EDUCATION AND TRAINING FOR WORK IN THE INDUSTRIALIZATION OF ANTIOQUIA - COLOMBIA}

\begin{abstract}
The Colombian industrialization process, immersed in the Import substitution industrialization -ISI-, took place between 1945 and 1980. It caused socio-technical changes in the country, mostly in highly industrialized areas such as the department of Antioquia and the surrounding areas of its capital city -Medellin-, Bello, Envigado, Itagüí -. Changes occurred at educational, social, economic, urbanistic, cultural and territorial levels.
\end{abstract}

Key words: industrialization - education - ISI Model - Antioquia 


\section{INTRODUCCIÓN:}

Desde la aparición de la industrialización en el siglo XVIII, primero en Europa y luego en otros lugares del mundo, existe el convencimiento de que esta es la forma socioeconómica más eficaz y eficiente de alcanzar el desarrollo económico de las sociedades modernas, por la creciente demanda de bienes y servicios (alimentos, bebidas, viviendas, vestido, salud, educación, leyes, etc.) capaces de satisfacer las necesidades básicas y suntuosas que, sin producción a nivel masivo, la humanidad sería incapaz de costear. A la par con el desarrollo económico, la industrialización conlleva, además, a transformaciones urbanas y modernización de los países y sus sociedades.

América Latina no fue ajena a la transformación económica y social, desde que entre finales del siglo XIX y primeras décadas del XX, se inició un proceso de cambio socioeconómico impulsado por el tránsito de un modelo de desarrollo agroexportador a uno industrial, con diverso ritmo según las condiciones iniciales de cada país; características estas, radicalmente diferentes al proceso de industrialización europeo y norteamericano de los siglos XVIII y XIX, pues la industrialización en la región se concibió desde el modelo de sustitución de importaciones -modelo ISI-, que a diferencia del modelo de industrialización clásico, avanza por etapas: inicialmente la fabricación de bienes de consumo primario, durables y no durables (sustitución fácil), para avanzar luego, sobre la base de acumulación de capital lograda en la primera etapa, a la siguiente que es de producción de bienes intermedios, y desembocar finalmente en la de fabricación de bienes de capital (sustitución difícil). Con la industrialización sustitutiva se esperaba que los países latinoamericanos alcanzaran en pocas décadas altas tasas de desarrollo económico sostenido, que se expresaran, a su vez, en una escalada del empleo que pudiera satisfacer la oferta creciente de mano de obra, resultado de la acelerada transición demográfica que caracterizó a las sociedades latinoamericanas en las primeras décadas del siglo XX.

El modelo ISI llegó a América Latina y a Colombia después de la Segunda Guerra Mundial y estuvo vigente entre 1945-1985, aproximadamente, momento a partir del cual el Estado colombiano toma las medidas necesarias para tratar de garantizar su éxito, creando las condiciones económicas, políticas y sociales necesarias y desarrollando una institucionalidad que favoreciera y fortaleciera el desarrollo económico y garantizara el progreso de la sociedad colombiana, mediante el aumento del empleo formal, una menor dependencia hacia los mercados extranjeros y el mejoramiento de los términos de intercambio comercial. El modelo ISI se convirtió en el paradigma de la modernización y del "desarrollo hacia adentro", al propiciar la producción en masa de grandes volúmenes de bienes y avanzar así por la senda del desarrollo y el bienestar social.

También se esperaba con la implementación del modelo ISI cambios a nivel social, como la expansión urbanística de las ciudades, elevar el nivel de vida de la población, obtener una mayor cobertura y expansión de la educación y de los servicios públicos, de salud, de comunicaciones y de transporte; aunque no podía preverse los cambios culturales y la adopción de valores más modernos, que llegaron a contraponerse a la tradición y al statu quo imperante por siglos. 

parcial de la investigación sobre.

\section{La industrialización en Antioquia- Colombia, 1945-1980: Cambio} sociotécnico y transformaciones sociales. Con este trabajo se optó al título de Magister en Estudios de Ciencia, tecnología, sociedad e innovación ${ }^{1}$. Se pretenden establecer aquí los cambios educativos que a nivel técnico y profesional se produjeron en Colombia, especialmente, en el departamento de Antioquia y su capital, Medellín, durante el período establecido.

\section{METODOLOGÍA}

En la investigación se adoptó el paradigma CTS, (estudios de Ciencia, tecnología y sociedad) y se recurrió a la técnica de recolección de datos de tipo investigación documental, en la que se buscó analizar información de fuentes secundarias para conocer, seleccionar y analizar aspectos históricos, contextuales, demográficos, organizacionales e institucionales. Ellas abundan en la literatura local y regional, tanto de la época, como de la actualidad: se asumen como tales para el presente artículo obras históricas, ensayos sociológicos, mapas, tablas y anuarios estadísticos del período en estudio, directorios telefónicos y fotografías.

El análisis de la información partió de la comprensión e interpretación históricosocial por medio de la investigación documental, buscando encontrar las aristas que permitieran la comprensión teórica e histórica del fenómeno. CTS constituye un paradigma alternativo de estudio para

\footnotetext{
1 Maestría en Estudios de CTS+i, del Instituto Tecnológico Metropolitano (ITM), en Medellín Antioquia (Colombia).
}

entender el fenómeno científicotecnológico en el contexto social. Se eligió el enfoque Tradicional Europeo que hace énfasis en los antecedentes de los factores sociales que influyen sobre el cambio científico-tecnológico.

\section{RESULTADOS:}

\section{La importancia de la educación y la capacitación para el trabajo en la Industrialización de Antioquia}

La educación colombiana en el siglo XX puede ser dividida en dos grandes momentos, correspondientes a la primera y segunda mitad del siglo. La primera mitad se caracterizó por un interés del Estado durante la vigencia de la llamada República Liberal, por mejorar la cobertura educativa, principalmente, en el sector de la educación primaria, pero sin alcanzar grandes logros a pesar del manifiesto interés. En la segunda parte del siglo se dio inicio al cambio de las políticas públicas en educación, llevándose a cabo la modernización del Ministerio de Educación Nacional, la creación del ICETEX (Instituto colombiano de especialización técnica en el exterior) en 1950, del SENA (Servicio nacional de aprendizaje) en 1957, y el instituto colombiano para el fomento a la educación superior (ICFES) en 1968, y con ello la creación y fomento de carreras técnicas y tecnológicas.

A inicios del siglo, en Antioquia, la industrialización comenzó a requerir mano de obra especializada, la cual no se podía esperar que resultase únicamente de la experiencia empírica de los trabajadores, sino que demandaba fuerza de trabajo calificada que solo podían proveer instituciones donde se 
capacitaran empresarios, técnicos y operarios, lo mismo que empleados dedicados al comercio y la banca para poder responder al proceso que se estaba gestando. Por esta razón, se reestructuraron los programas académicos y se crearon instituciones especializadas.

\section{Como bien afirma Saavedra:}

La formación académica como propósito deliberado debía ceder el paso a una formación más asociada con lo práctico, no solo se trataba del interés por una instrucción académica formal a la que no todos tenían acceso, sino que se trataba del impulso a estudios técnicos y a la capacidad práctica, acordes con las necesidades inmediatas de la región (Saavedra, 1992, pág. 109).

Lo anterior explica que desde finales del siglo XIX se hubieran comenzado a crear este tipo de instituciones, orientadas por el ideal de lo práctico, como la Escuela de Artes y Oficios, que había sido creada a finales del siglo XIX con la finalidad de armonizar la teoría y la práctica, no solo en el aula sino en el taller, por lo que la escuela era un taller manufacturero, al estilo de las escuelas de artes y oficios francesas, que combinaba los oficios tradicionales con los modernos (construcción, mecánica, arquitectura) y fue la que cimentó los posteriores estudios de ingeniería con la implementación de la enseñanza de las "matemáticas modernas superiores" para dominar el entorno natural y poner la energía hidráulica y la hidroeléctrica al servicio de la comunidad. (Mayor, 2013, pág. 23).

La Escuela de Minas, simiente de la Universidad Nacional, sede Medellín, fue creada a finales del siglo XIX; inicialmente, la primera encargada de instruir ingenieros para la minería y, después, en la industria cuando el proceso de crecimiento industrial era notorio y había necesidad de capacitación:

Por muchos años, la Escuela sólo tuvo el programa para la formación de Ingenieros Civiles y de Minas. Más tarde, entre 1941 y 1950, diversas disposiciones dieron origen a que las carreras ofrecidas por la Facultad de Minas fuesen las de Ingeniería

Civil, Ingeniería de Minas y Metalurgia, Ingeniería Geológica y Petróleos y Arquitectura; esta última se separó de la Facultad en 1954. Posteriormente se creó la carrera de Ingeniería Administrativa, en 1960. Más adelante se crearon los programas de Ingeniería Industrial, Ingeniería Mecánica e Ingeniería Química y se separó Geología y Petróleos en dos carreras diferentes... (http://minas.medellin.unal.edu.co/lafacultad/ historia, 2015)

Los primeros egresados de la Escuela de Minas fueron llevados a la empresa pública "para que acrisolara el ideal de servicio público a la comunidad a través de un manejo eficiente y una mayor productividad y, luego a la empresa privada, donde aquellas virtudes civiles se multiplicaran de modo extraordinario en función de los intereses privados.” (Mayor, 2013, pág. 151). Podría preguntarse, entonces, si las empresas públicas fueron usadas como conejillos de indias para que los aprendices tomaran la experiencia que luego usarían para multiplicar los intereses privados. En otros términos ¿Era entonces lo público usado como experimentación en beneficio de las élites industriales recién creadas sin importar que la inexperiencia llevara a lo público al colapso económico?

En 1936 se creó la Universidad Católica Bolivariana, como respuesta a las nuevas políticas liberales, pues muchos profesores y estudiantes conservadores consideraban 
Albenis María Monterroza Ríos

El aporte de la educación y la capacitación para el trabajo en la industrialización de Antiquia - Colombia págs. 19- 29

que eran discriminatorias y represivas hacia ellos. Esta nueva universidad comenzó a funcionar con las facultades de Derecho y Medicina ${ }^{2}$ y en 1938 entró en funcionamiento la primera carrera de Ingeniería Química del país, por la necesidad que había de formar ingenieros para las grandes textileras que se perfilaban en la ciudad.

En el mismo año se formó el "círculo de estudios para obreros” (Aguilar, 2006, pág. 12).

La universidad tuvo mucha acogida y siguió creciendo con una fuerte demanda. En 1942 se formó la Facultad de Arquitectura, segunda del país y primera de Antioquia, la cual dio un gran impulso al creciente urbanismo de la ciudad. En 1943 la Facultad de Arte y Decorado da paso a la posterior Facultad de Diseño. En 1950 se funda la Facultad de Ingeniería Eléctrica, en 1956 la de Ingeniería Mecánica y el Instituto de Ciencias Pedagógicas y Sociales - hoy Facultad de Educación y Pedagogía-, y en 1959 la de Sociología. Fueron creadas posteriormente las facultades de teología, comunicación social, ingeniería electrónica, psicología, enfermería, publicidad, ingeniería agroindustrial, ingeniería textil, ingeniería informática y telecomunicaciones, ingeniería aeronáutica, ingeniería industrial y se reabriría medicina que solo había funcionado un año inicialmente (Aguilar, 2006).

La formación y requerimientos de los nuevos dirigentes de las diferentes actividades empresariales de finales de los años cincuenta, creó la necesidad de capacitar individuos con una educación universitaria acorde a los nuevos retos de las empresas y avances en la enseñanza que permitiera a los dirigentes mejores competencias técnicas y administrativas. Así afirma Restrepo:

[...] a partir de 1960 se crearon nuevos centros universitarios para impartir educación especializada, se mejoró la formación de ejecutivos mediante programas de extensión y cursos de administración, se estimularon los programas de capacitación interna y se acudió al reclutamiento de empleados más calificados (Restrepo, 2011, pág. 84).

En este sentido la Universidad Pontificia Bolivariana, como pasó a llamarse en 1945, se convirtió, junto con la Universidad de Antioquia y la Facultad de Minas (luego Universidad Nacional de Colombia seccional Medellín), en uno de los principales baluartes de la educación tecnológica y profesional de la región.

Como se puede apreciar en la tabla 1, la educación se fue diversificando y cada vez hubo más estudiantes en busca de llenar esos vacíos en la formación industrial y comercial de la región, gracias a la implementación de nuevas carreras técnicas, tecnológicas y de nivel superior en establecimientos tanto públicos como privados, que permitieron continuar con la profesionalización de la mano de obra.

Anuario Estadístico de Antioquia entre 1945 y 1980.

\footnotetext{
2 Si bien en esta fecha se creó la facultad de medicina, solo duró un año. En realidad, esta facultad comenzó a existir apenas desde febrero de 1976. Es decir, casi cuarenta años después.
} 
TABLA 1: Establecimientos educativos y estudiantes matriculados en Antioquia

\section{$1961-1978$}

\begin{tabular}{|c|c|c|c|c|}
\hline \multirow[b]{2}{*}{ CLASES DE ENSEÑANZAS } & \multirow[b]{2}{*}{ No Establecimientos } & \multicolumn{3}{|c|}{ ALUMNOS MATRICULADOS } \\
\hline & & Hombres & Mujeres & Total \\
\hline \multicolumn{5}{|c|}{1961} \\
\hline Comercial Oficial & 4 & 282 & 874 & 1156 \\
\hline Comercial privada & 21 & 411 & 1576 & 1987 \\
\hline Industrial oficial & 8 & 2147 & 689 & 2836 \\
\hline Industrial privada & 3 & 419 & 3 & 422 \\
\hline Subtotal & 36 & 3259 & 3142 & 6401 \\
\hline \multicolumn{5}{|c|}{1962} \\
\hline Comercial Oficial & 4 & 169 & 875 & 1044 \\
\hline Comercial privada & 19 & 377 & 1455 & 1832 \\
\hline Industrial oficial & 9 & 2187 & 657 & 2844 \\
\hline Industrial privada & 5 & 432 & & 432 \\
\hline Subtotal & 37 & 3165 & 2987 & 6152 \\
\hline
\end{tabular}

1963

\begin{tabular}{|c|c|c|c|c|}
\hline Comercial Oficial & 3 & & & \\
\hline Comercial privada & 15 & & & \\
\hline Industrial oficial & 9 & & & \\
\hline Industrial privada & 3 & & & \\
\hline \multirow[t]{2}{*}{ Subtotal } & 30 & & & \\
\hline & 1964 & & & \\
\hline Comercial Oficial & 4 & 516 & 1093 & 1609 \\
\hline Comercial privada & 15 & 332 & 1278 & 1610 \\
\hline Industrial oficial & 9 & 1721 & 379 & 2100 \\
\hline Industrial privada & 3 & 362 & & 362 \\
\hline Subtotal & 31 & 2931 & 2750 & 5681 \\
\hline
\end{tabular}

REVISTA CIENCIA, CULTURA Y SOCIEDAD 


\begin{tabular}{|c|c|c|c|c|}
\hline & 1965 & & & \\
\hline Comercial Oficial & 4 & 484 & 1224 & 1708 \\
\hline Comercial privada & 12 & 410 & 936 & 1346 \\
\hline Industrial oficial & 9 & 1938 & 231 & 2169 \\
\hline Industrial privada & 3 & 392 & & 392 \\
\hline \multirow[t]{2}{*}{ Subtotal } & 28 & 3224 & 2391 & 5615 \\
\hline & 1972 & & & \\
\hline \multicolumn{2}{|l|}{ Técnica industrial oficial } & & 384 & 384 \\
\hline Técnica industrial privada & 10 & 3506 & 1068 & 4574 \\
\hline Superior & 112 & & & 25989 \\
\hline Artes y oficio y economía oficial & 20 & 573 & 3564 & 4127 \\
\hline Artes y oficio y economía privada & 4 & 39 & 204 & 243 \\
\hline Sena oficial & 1 & & & 52128 \\
\hline
\end{tabular}

1973

\begin{tabular}{|c|c|c|c|c|}
\hline Técnica industrial oficial & 10 & & & \\
\hline Técnica industrial privada & 2 & & & \\
\hline Superior & 154 & & & \\
\hline Artes y oficio y economía oficial & 23 & & & \\
\hline Artes y oficio y economía privada & 2 & & & \\
\hline \multirow[t]{2}{*}{ Sena oficial } & 5 & & & \\
\hline & 1974 & & & \\
\hline \multicolumn{2}{|l|}{ Comercial oficial } & & 424 & 424 \\
\hline \multicolumn{2}{|l|}{ Comercial privada } & 20 & 17 & 37 \\
\hline Técnica Industrial oficial & 10 & 3347 & 1264 & 4611 \\
\hline Técnica industrial privada & 2 & 816 & 4 & 820 \\
\hline \multicolumn{2}{|l|}{ Media diversificada oficial } & 2465 & 1191 & 3656 \\
\hline Superior & 16 & & & 26107 \\
\hline Universitario oficial & 5 & & & 12507 \\
\hline Universitario privado & 11 & & & 12581 \\
\hline Capacitación comercial privada & 26 & 1615 & 5192 & 6807 \\
\hline Sena oficial & 5 & & & 52789 \\
\hline
\end{tabular}

REVISTA CIENCIA, CULTURA Y SOCIEDAD 


\begin{tabular}{lccccc}
\hline Bachillerato, Normalista y comercial & & 364 & 92892 & 95699 & 188591 \\
\hline Técnica Industrial & 11 & 4507 & 1565 & 6072 \\
\hline & 1976 & & & &
\end{tabular}

\begin{tabular}{|c|c|c|c|c|}
\hline Bachillerato, Normalista y comercial & 380 & 102028 & & 211268 \\
\hline Técnica Industrial & 10 & 4893 & & 6136 \\
\hline Privada Técnica industrial & 1 & 687 & & 687 \\
\hline Superior & 17 & & & 36820 \\
\hline Oficial & 5 & & & 22429 \\
\hline Carreras de nivel medio & & & & 910 \\
\hline Universitario & 5 & & & 21519 \\
\hline Privada & 12 & & & 14391 \\
\hline Carreras de nivel medio & & & & 337 \\
\hline Universitario & 12 & & & 14054 \\
\hline Otras enseñanzas & 374 & & & 121760 \\
\hline Oficial & 320 & & & 106859 \\
\hline \multirow[t]{2}{*}{ Sena } & 1 & & & 64333 \\
\hline & 1977 & & & \\
\hline Bachillerato, Normalista y comercial & 392 & 107247 & 118775 & 226022 \\
\hline Técnica Industrial & 8 & 4888 & 135 & 5023 \\
\hline Privada Técnica industrial & 2 & 706 & 19 & 725 \\
\hline Superior & 17 & & & 38284 \\
\hline Oficial & 5 & & & 22945 \\
\hline Carreras de nivel medio & & & & 511 \\
\hline Universitario & 5 & & & 22434 \\
\hline Privada & 12 & & & 15339 \\
\hline Carreras de nivel medio & & & & 429 \\
\hline Universitario & 12 & & & 14910 \\
\hline Otras enseñanzas & 321 & & & 153338 \\
\hline Oficial & 251 & & & 127252 \\
\hline Sena & 1 & & & 88213 \\
\hline
\end{tabular}




\begin{tabular}{lcccc}
\hline \multicolumn{1}{c}{1978} & & & \\
\hline Bachillerato, Normalista y comercial & 421 & 108897 & 129683 & 238580 \\
\hline Técnica Industrial & 9 & 5331 & 145 & 5476 \\
\hline Privada Técnica industrial & 2 & 606 & 606 \\
\hline Superior & 18 & 42162 \\
\hline Oficial & 5 & 24008 \\
\hline Carreras de nivel medio & 5 & 142 \\
\hline Universitario & 13 & 23866 \\
\hline Privada & 1 & 18154 \\
\hline Carreras de nivel medio & 12 & 760 \\
\hline Universitario & 245 & 17394 \\
\hline Otras enseñanzas & 205 & 138369 \\
\hline Oficial & 1 & 96815 \\
\hline Sena & & & 124443 \\
\hline Fuente: Elaborán & & & \\
\hline
\end{tabular}

Fuente: Elaboración propia basada en datos estadísticos

En la misma Tabla 1, se puede observar cómo el SENA va tomando mayor auge desde su creación en 1957, y entra a jugar un papel importante en la formación de los técnicos que se necesitaban en la industria local. Esta institución ofrecía una formación de carácter teórico y, a la vez, práctico con la pretensión de brindar mano de obra calificada con métodos modernos capaces de generar cambios en la productividad e idóneo para la vinculación inmediata a las empresas. Entre las funciones definidas en su decreto de formación se encuentra la de "... brindar formación profesional a los trabajadores, jóvenes y adultos de la industria, el comercio, la agricultura, la minería y la ganadería" (Centro Virtual de Noticias de la Educación, 2015). En esta entidad se hacen partícipes los trabajadores, empleadores y el gobierno. En sus inicios se ofrecían en las zonas urbanas las carreras de construcción, secretariado y finanzas; mientras que en las zonas rurales, la enseñanza se centraba en la agricultura y su tecnificación.

Posterior a su año de creación se llevó a cabo un estudio que pretendía conocer de primera mano las necesidades de las empresas en cuanto a la formación profesional de sus empleados, para diversificar la oferta de nuevos cursos destinados a ese fin:

En 1958 se realizó una investigación en cinco mil empresas del país acerca de las necesidades de formación profesional. El estudio abarcó todos los sectores de la industria y reveló que al menos 210 mil trabajadores requerían complementar su educación y urgía la formación de 25 mil trabajadores adicionales. Con base en estos resultados se fijó el plan quinquenal, 19591963. (Centro Virtual de Noticias de la Educación, 2015). 
En 1960 el SENA firmó convenio con la OIT (Organización Internacional del Trabajo) para llevar a cabo programas de asesoría financiera, administrativa y técnica con pequeños y medianos empresarios, en este marco se realizó el estudio titulado "Investigación de Recursos Humanos para una Política Nacional de Empleo y Formación Profesional", que representó el punto de partida para llegar a las empresas con una mejor oferta de capacitación y mejoramiento de la demanda de cursos necesarios para el desarrollo de las mismas

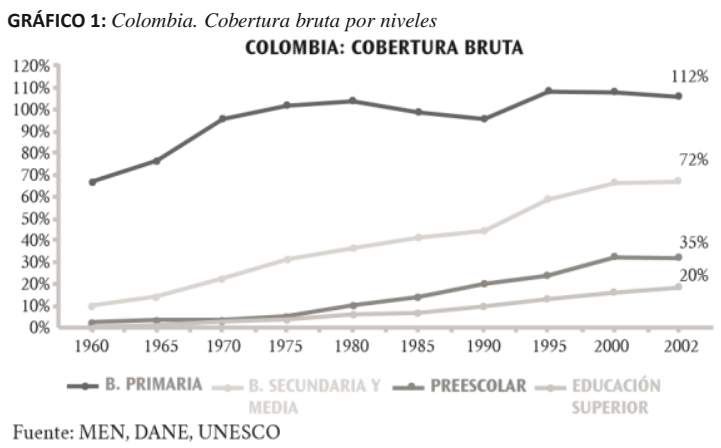

Tomado de: Políticas educativas Colombia. Plan sectorial 20022006.

El gráfico 1 muestra la escasa cobertura de la educación superior, en la segunda mitad del siglo $\mathrm{XX}$, con respecto a la población con cobertura en Educación Básica Primaria, es decir, que muchas personas no pasaban del nivel básico de la educación y esta cantidad de escolarizados iba disminuyendo a medida que avanzaban en los estudios. Como se observa en el citado gráfico, para 1980 la educación superior apenas llegaba al $10 \%$ de cobertura.

\section{CONCLUSIONES:}

Desde el punto de vista sociocultural, la industrialización antioqueña estimuló la apertura de nuevos centros educativos $\mathrm{y}$

REVISTA CIENCIA, CULTURA Y SOCIEDAD

Vol. 5, n. ${ }^{\circ}$ 1, enero-junio/ 2018. ISSN 2305-7688 formativos que permitieron la capacitación de mano de obra para laborar en las empresas y asumir los retos técnicos y empresariales que planteaba la industrialización, con efectos positivos en la productividad y la competitividad. Es decir, la educación jugó un papel importante en la formación de nuevas actitudes para asumir los retos técnicos y empresariales que se crearon con la industrialización.

La creación de nuevas universidades y centros de educación superior y de capacitación técnica, diferentes o nacidas del seno de las ya existentes, fue otro hito importante (la Facultad de Minas, Universidad Pontificia Bolivariana, Eafit, y el SENA, entre otras) pues estas instituciones contribuyeron significativamente a la formación de cuadros técnicos y profesionales, urbanísticos y sociales que demandaban las empresas tanto industriales como de servicios. La creación de carreras humanísticas y pedagógicas fue un gran aporte, debido a que permitieron estudiar y comprender el fenómeno social por el que estaba atravesando la región, creando con ello un espíritu crítico y ajustado a los cambios sociales.

Entre las principales conclusiones, debemos anotar la desproporción entre la cantidad de estudiantes de la Educación Básica Primaria y la Educación Universitaria, que permite ver la inequidad social que se gestaba en el acceso a la educación y que, a la vez, no le dejaba a la industria contar con la mano de obra necesaria para afrontar todos los retos del mejoramiento tecnológico y la expansión industrial. 


\section{FUENTES CONSULTADAS}

Aguilar, R. (2006). Universidad Pontificia Bolivariana. 70 años (1936-2006). Historias Contadas, 11-13.

Arango, M. (1990). Los Funerales de Antioquia La Grande. La Bancarrota del Modelo Económico Antioqueño. Medellín: J.M.Arango.

Centro Virtual de Noticias de la Educación. (16 de Agosto de 2015). http://www.mineducacion.gov.co/cvn/1665/ w3article-307852.html. Obtenido de http://www.mineducacion.gov.co/cvn/1665/w3article-307852.html.

Gaviria, J. F. (1968). La Industria en Antioquia. Revista de la ANDI, 3-48.

González, L. (2007). Medellín, los orígenes y la transición a la modernidad: crecimiento y modelos urbanos. 1775-1932. Medellín: Escuela del HábitatUniversidad Nacional. http://minas.medellin.unal.edu.co/lafacultad/historia. (12 de Agosto de 2015).

Mayor, A. (2005). Inventos y Patentes en Colombia 1930 - 2000. Medellín: Instituto Tecnológico Metropolitano.

Mayor, A. (2013). De la energía de Prometeo a la forja de Vulcano. El ingeniero Julián Cock Arango, un héroe del trabajo en Antioquia, 1982-1982. Medellín. Fondo Editorial ITM.

Monterroza, A. (2017). La industrialización en Antioquia-Colombia, 1945-1980: Cambio sociotécnico y transformaciones sociales. Medellín.

Ocampo T., J. F. (1999). Elementos para una historia de la educación pública colombiana en la segunda mitad del siglo $x x$ : luces y conquistas, sombras $y$ engendros: 19502000. Revista Educación y Cultura.

Poveda, G. (1967). Población, industrialización y empleo. Revista de la ANDI, 3 - 29.

Restrepo, N. (2011). Empresariado antioqueño y sociedad, 1940-2004. Medellín: Editorial Universidad de Antioquia.

Saavedra, M. (1992). Antioquia en los inicios del proceso de industrialización: algunos aspectos relativos a la capacitación técnica. Lecturas de Economía No 37, 107-126. e 